\section{OP0123 PREDICTION OF PROGRESSIVE SKIN THICKENING IN EARLY DIFFUSE SYSTEMIC SCLEROSIS USING THREE-MONTHLY SKIN SCORES FROM THE EUROPEAN SCLERODERMA OBSERVATIONAL STUDY (ESOS)}

A. Herrick ${ }^{1}$, S. Peytrignet ${ }^{1}$, X. Pan ${ }^{1}$, R. Hesselstrand ${ }^{2}$, L. Mouthon ${ }^{3}$, E. Brown ${ }^{1}$ L. Czirják ${ }^{4}$, J.H. Distler ${ }^{5}$, O. Distler ${ }^{6}$, K. Fligelstone $^{1}$, W. Gregory ${ }^{1}$, R. Ochiel ${ }^{7}$, A. Silman ${ }^{8}$, M. Vonk ${ }^{9}$, M. Lunt ${ }^{1}$, C. Denton ${ }^{7} .{ }^{1}$ University of Manchester, Manchester, United Kingdom; ${ }^{2}$ Lund University, Lund, Sweden; ${ }^{3}$ Université Paris Descartes, Paris, France; ${ }^{4}$ University of Pécs, Pécs, Hungary; ${ }^{5}$ University of Erlangen-Nuremberg, Erlangen, Germany; ${ }^{6}$ University Hospital Zurich, Zurich, Switzerland; ${ }^{7}$ University College London, London; ${ }^{8}$ University of Oxford, Oxford, United Kingdom; ${ }^{9}$ Nijmegen University, Nijmegen, Netherlands

Background: ESOS (European Scleroderma Observational Study) was a prospective observational study of early diffuse cutaneous systemic sclerosis, recruiting from 50 centres in 19 countries and thus providing a unique opportunity to study parameters of disease progression at regular intervals.

Objectives: To describe the characteristics of patients with progressive skin thickening and derive prediction models for progression over 12 months.

Methods: Duration of skin thickening and autoantibody status (antitopoisomerase-1[anti-Scl-70, TOPO], anti-RNA polymerase III[Pol3], anticentromere[ACA]) were documented. The modified Rodnan skin score (mRSS) was recorded 3-monthly for up to two years after baseline. The main outcome was the progression of mRSS. Progressive patients ("progressors") had to meet a 5-unit and 25\% increase in their mRSS within the first 12 monthsof follow-up. Features of progressors vs. non-progressors were compared using the Fisher or Kruskal-Wallis test (for categorical and continuous variables), as were progression parameters between autoantibody groups. Logistic models were fitted to predict progression and, using ROC curves, were compared based on AUC, accuracy and positive predictive value (PPV).

Results: 326 patients were recruited, with median disease duration of 11.9 months. During the first 12 months, 66 patients (20.3\%) progressed, 227 (69.6\%) did not and $33(10.1 \%)$ could not have their status assessed. At baseline, progressors had shorter disease duration than non-progressors: 8.1 months vs. 12.6 months $(\mathrm{p}=0.001)$. Progressors started with a lower mRSS, median 19 units vs. 21 for non-progressors $(\mathrm{p}=0.030)$.

124 patients were $\mathrm{TOPO}+, 50$ were $\mathrm{Pol} 3+, 20$ were $\mathrm{ACA}+, 2$ were TOPO+/ACA+ and 68 had none. Pol3+ patients had a higher mRSS peak (35 units vs. 27 overall[ $[=0.001]$ ) and did so earlier (median 17.9 months vs. 23.1 months overall $[p=0.214])$

Using an mRSS 22 cutoff point to predict progression in the ESOS cohort (as suggested in the literature) would yield a PPV of $24.3 \%$, a weak improvement from the observed $20.3 \%$ share of progressors. A first model (Model A, with mRSS, duration of skin thickening and their interaction) had an accuracy of $60.9 \%$, AUC of 0.67 and PPV of $33.8 \%$. Figure 1summarizes the prediction rule, with patients under the curved line predicted to progress. By adding a variable for being Pol3+ (Model B), the model reached an accuracy of $71 \%$, AUC of 0.71 and PPV of $41 \%$.

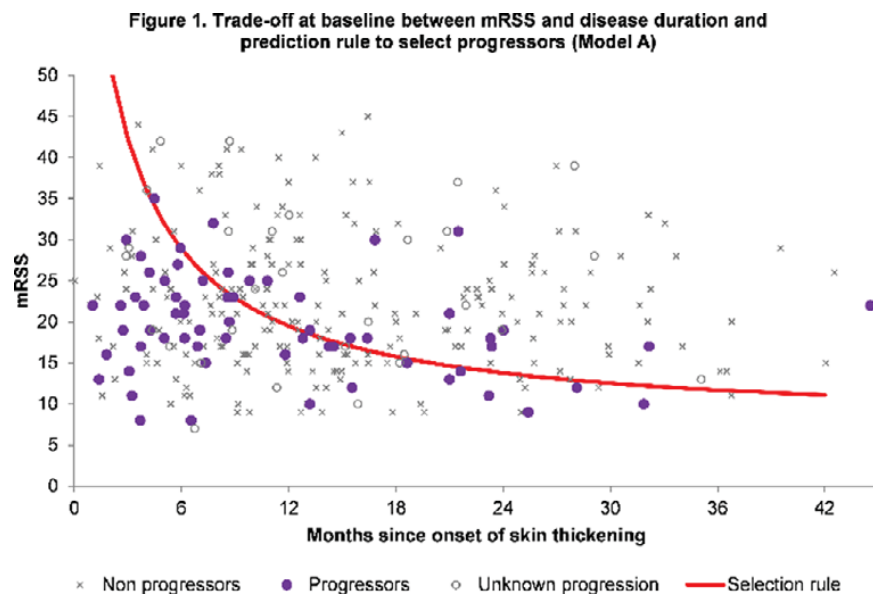

Conclusions: 1. Patients with shorter disease duration and a lower mRSS have a higher likelihood of being progressors, with a trade-off between the two.

2. Pol3+ patients experience higher mRSS peaks and tend to reach them earlier.

3. Two prediction models for progressive thickening were derived. The advantage of having two is that Model $\mathrm{B}$, while more accurate and useful in identifying high-risk patients in clinical practice, risks being too restrictive for patient selection into trials and may over-represent Pol3+ patients.

Disclosure of Interest: None declared

DOI: 10.1136/annrheumdis-2017-eular.1869

\section{OP0124 TREATMENT WITH CYCLOPHOSPHAMIDE FOR SYSTEMIC SCLEROSIS-RELATED INTERSTITIAL LUNG DISEASE DOES NOT IMPROVE SURVIVAL AFTER 12 YEARS OF FOLLOW UP}

E.R. Volkmann ${ }^{1}$, M. Sim ${ }^{1}$, D.P. Tashkin ${ }^{1}$, D. Khanna ${ }^{2}$, P.J. Clements ${ }^{1}$, M. Roth ${ }^{1}$, D. Furst ${ }^{1}$, A. Pickney ${ }^{3}$, E. Goldmuntz ${ }^{4}$, L. Keyes-Elstein ${ }^{3}$,

R. Elashoff ${ }^{1}$, K. Sullivan ${ }^{5}$ on behalf of SLS I working group. ${ }^{1}$ University of California, Los Angeles, Los Angeles; ${ }^{2}$ University of Michigan, Ann Arbor; ${ }^{3}$ Rho Federal Systems, Inc., Chapel Hill; ${ }^{4}$ DAIT, Niaid/Nih, Bethesda; ${ }^{5}$ Duke University, Durham, United States

Background: Treatment with cyclophosphamide (CYC) is associated with shortterm improvements in lung function, dyspnea, and radiographic fibrosis in patients with systemic sclerosis-related interstitial lung disease (SSc-ILD). ${ }^{1}$ However, the effects of CYC therapy on long-term morbidity and mortality outcomes are unknown.

Objectives: To determine whether 1 year of treatment with CYC for SSc-ILD affects long-term morbidity and mortality in patients who participated in the Scleroderma Lung Study (SLS) I. ${ }^{1}$

Methods: SLS I randomized 158 SSc-ILD patients from 13 US SSc centers to 1 year of oral CYC versus placebo. The primary endpoint was the change in FVC\% $>$ predicted over 1 year. Twelve years after the study commenced, each study center contacted enrolled patients or designated surrogates to assess the following: mortality, cause of mortality, development of organ failure, need for transplant and functional status. We used counting process cox proportiona hazard modeling to determine the variables associated with survival. The model findings were validated using a joint model of longitudinal and survival data. We also tested the model using long-term follow-up data from SLS II (CYC vs. Mycophenolate).

Results: Nearly half of all SLS I patients (43\%) died during the follow-up period, and only $24 \%$ remained alive in the absence of organ failure. The median follow up period for all patients was 8 years. Where known, the cause of death was attributable most often to SSc. Among patients who developed malignancy $(\mathrm{N}=13)$, a similar proportion were treated with $\mathrm{CYC}$ compared with placebo. The most common type of organ failure was respiratory failure $(\mathrm{N}=31$ of 33 organ failures) defined as the need for supplemental oxygen therapy $(\mathrm{N}=29)$ and/or lung transplantation $(\mathrm{N}=3)$. There was no difference in the time to death (Figure 1), or time to organ failure, or time to malignancy in patients randomized to CYC versus placebo. The Cox model identified the following variables as the most important predictors of mortality: baseline skin score (HR 1.033; $P=0.0038$ ), age at randomization (HR 1.056; $P<0.0001$ ), and the course of the FVC from baseline to 24 months (HR 0.975; $P=0.0215$ ). The course of the FVC was a better predicto of mortality than the baseline FVC. The joint model identified the same variables associated with mortality.

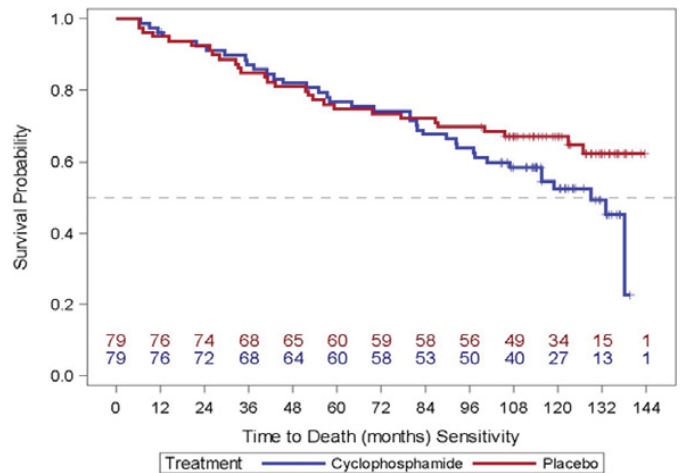

Figure 1. Time to death in patients randomized to CYC (blue line) and placebo (red line).

Conclusions: Treatment with 1-year of oral CYC for SSc-ILD does not decrease long-term mortality, organ failure or malignancy compared with placebo. In addition to identifying traditional mortality risk factors in SSc (i.e. increased skin score and advanced age), this study found that progression of the FVC over 2 years was a more important predictor of mortality than the baseline FVC. These findings suggest that early changes in surrogate measures of SSc-ILD progression may have important effects on long-term outcomes.

References:

[1] Tashkin et al. NEJM 2006;354:2655

Disclosure of Interest: None declared

DOI: 10.1136/annrheumdis-2017-eular.2488

\section{OP0125 MORTALITY IN PATIENTS WITH DERMATOMYOSITIS/POLYMYOSITIS IN A CHINESE MEDICAL} CENTRE

X. Yang. Peking University First Hospital, Beijing, China

Objectives: To investigate the mortality and the causes of death in Chinese patients with dermatomyositis (DM) or polymyositis (PM).

Methods: We collected the clinical data of all DM/PM patients in Rheumatology 